\section{E.5 SHORT-TERM DISABILITY LEAVE AND EMPLOYMENT TERMINATION: USING MARGINAL STRUCTURAL MODELS TO ESTIMATE COUNTERFACTUAL RISKS}

${ }^{1}$ Sally Picciotto*, 'Andreas Neophytou, ${ }^{2}$ Mark Cullen, ${ }^{1}$ Ellen Eisen. ${ }^{1}$ UC Berkeley, Berkeley, USA; ${ }^{2}$ Stanford University, Palo Alto, USA

\subsection{6/OEM-2019-EPI.31}

Introduction Short-term disability leave can be considered as a measure of not being well enough to work. The American Manufacturing Cohort, followed 1996-2013, consists of employees of a light-metal company that provided short-term disability insurance to all employees: coverage to replace wages for up to 6 months of work absence due to medical issues. We hypothesized that since brief short-term disability leave allows workers time to recover from illness or injury without losing their jobs, it should be protective against employment termination.

Methods We analyzed $18386 \quad$ (83\% male, 80\% white) hourly employees. We censored workers once their accumulated disability leave exceeded 6 weeks because longer time spent on short-term disability leave suggests more serious illness or injury that may prevent return to work. To analyze the effect of short-term disability leave on employment termination, we applied a marginal structural pooled logistic model that allowed for a time-varying hazard function. We adjusted for time-varying confounding by occupational exposures and health-related variables using inverse probability weighting. Using the estimated coefficients, we compared the predicted probabilities (by person-month) of terminating employment with the corresponding counterfactual probabilities if the worker had never taken disability leave. These probabilities yielded estimated survival curves under the two scenarios.

Results The average worker was followed for 5.5 years. Approximately $42 \%$ of the workers took at least one day of disability leave, and 48\% terminated employment during follow-up. We estimated that 1058 (29\%) more workers would have terminated employment within 5 years from cohort entry if the company had had no disability leave benefit than were predicted under the natural course.

Conclusion Short-term disability leave is a potentially relevant health variable for occupational epidemiologists. This analysis suggests that short-term disability leave can help employees retain their jobs when a temporary health issue prevents them from working.

\section{Work Organisation}

\section{E.6 THE IMPACT OF A PROGRAM OF MANDATORY AWARENESS TRAINING ON WORKER AWARENESS AND EMPOWERMENT TO PARTICIPATE IN INJURY PREVENTION}

Peter Smith*. Institute for Work and Health, Toronto, Canada

\subsection{6/OEM-2019-EPI.32}

On July 1 st 2014, the province of Ontario introduced a program of mandatory occupational health and safety (OHS) awareness training. The objective of this study was to evaluate the impact of this program on worker level self-reported
OHS awareness and empowerment to participate in injury prevention activities in Ontario, compared to the province of British Columbia (BC) where no similar program was introduced.

We collected information on OHS awareness and empowerment, as well as participation in OHS training from samples of workers in Ontario and BC on four occasions. The first survey was conducted between May and June 2014 (prior to the introduction of mandatory training); then additional surveys were completed in February/March 2015, September/ October 2015, and November/December 2017. For each cross-sectional sample we recruited over 1800 respondents, with a total sample of over 7500 respondents $(63 \%$ from Ontario) across all time points. Logistic regression models were used to examine a time by province interaction in each outcome, after adjusting for a variety of workplace and worker characteristics.

After the introduction of mandatory awareness training respondents in Ontario were almost twice as likely to participate in training compared to respondents in BC. However, we observed no difference in trends over time (pre-post introduction of mandatory training) in levels of adequate awareness or empowerment between Ontario and BC. Respondents in Ontario had higher levels of adequate OHS awareness than respondents in $\mathrm{BC}$ at all time points (both before and after the introduction of mandatory awareness training). No differences were present between Ontario and $\mathrm{BC}$ in relation to empowerment.

Taken together, these results suggest that the introduction of mandatory awareness training in Ontario was associated with greater training participation. However, no differences in levels of adequate awareness or empowerment were observed between Ontario respondents compared to $\mathrm{BC}$ respondents.

\section{Pesticide Health Effects}

\section{O2A.1 PESTICIDES AND WORK-RELATED ASTHMA: HOW THIS RELATES TO SELF-REPORTED EXPOSURES}

'David Fishwick*, 'Anne-Helen Harding, 'David Fox, ${ }^{1}$ Yiqun Chen, ${ }^{2}$ Neil Pearce, ${ }^{1}$ Gillian Frost. ${ }^{1}$ HSE GB and NHS, Sheffield, UK; ${ }^{2}$ London School of Hygiene and Tropical Medicine, London, UK

\subsection{6/OEM-2019-EPI.33}

Pesticide exposure has been linked to a number of potentially adverse health outcomes, including asthma. We were interested to explore the relationship between asthma and pesticide use, particularly from the perspective of self-reported work-related asthma.

Using the existing UK PIPAH (Prospective Investigation of Pesticide Applicators' Health) study population, we administered a respiratory questionnaire to 4814 current and past pesticide users. Participants were asked about doctor-diagnosed and self-reported asthma symptoms, and, if present, their views about the cause of work-related asthma (asthma reported to be worse at work).

Of the 2562 respondents (53\% response) with a median age of 60.2 years, $97.4 \%$ were male and $34.1 \%$ ever smoked. The prevalence of ever being doctor diagnosed asthma was $11.4 \% \quad(n=292)$, and 123 of these $(42.1 \%$ of those with 